\title{
Comparison of clinical outcome between pyeloperfused versus non-pyeloperfused microwave ablation of renal cell carcinoma
}

\author{
Katayoun Samadi ${ }^{1 A, B, C, D, E, E, G}$, Ronald Arellano $2 \mathrm{AB,B}, \mathrm{CD}, \mathrm{E}, \mathrm{F}$ \\ 'Department of Interventional Radiology, Massachusetts General Hospital, Boston, MA, United States \\ 2Department of Radiology, Division of Interventional Radiology, Massachusetts General Hospital, Boston, MA, United States
}

\section{Abstract}

Purpose: We present the outcomes of microwave ablation (MWA) of renal cell carcinoma (RCC) with and without pyeloperfusion.

\begin{abstract}
Material and methods: A retrospective review of patients' records was undertaken to identify patients with RCC, who were treated with MWA with and without adjunctive pyeloperfusion. The distance between the tumour and ureter as well as the tumour size were measured on axial imaging. Pyeloperfusion was performed in nine patients in this series after placement of a ureteral stent and instilment of diluted contrast into the ureter. MWAs of the tumours were performed under computed tomography (CT) guidance. Hydrodissection was performed to displace at-risk organs. Creatinine was measured as renal function index after and before the procedure. A CT scan was performed at the end of the procedure and also after one, three, and six months, to identify the presence of residual disease and complications.

Results: Eighteen biopsies of proven RCC were treated with 20 sessions of MWA. The average follow-up time for this study was 180 days. The average distance between the ureter and the tumour in axial CT view was $20.8( \pm 2.9) \mathrm{mm}$. Primary efficacy was achieved in $88 \%$ of pyeloperfused patients and in $100 \%$ of the non-pyeloperfused patients. Two pyeloperfused patients required secondary procedure, and full secondary efficacy was achieved for both. There was only one grade 2 urological complication, which occurred in a patient who underwent pyeloperfusion. Creatinine was not significantly different after the procedure in this study ( $p$-value 0.4 ).
\end{abstract}

Conclusion: In this study MWAs of RCCs were successfully performed using pyeloperfusion as a protective measure against thermal injury to the ureter.

Key words: microwave ablation, pyeloperfusion, hydrodissection.

\section{Introduction}

Advances in imaging modalities and diagnostic techniques have led to an increase in the detection and diagnosis of renal cell carcinoma (RCC) [1]. While partial nephrectomy is considered the gold standard treatment for RCCs, ablative therapies such as radiofrequency ablation (RFA), cryoablation (CA), and microwave ablation (MWA) are alternatives to nephrectomy in selected patients for whom surgery may not be an option [2]. Of the three currently available thermal ablative options RFA and CA are the most extensively investigated, and several studies have demonstrated similar clinical efficacies for RFA and CA for the management of small renal cell tumours $[3,4]$.

MWA is the latest technique and may offer the benefits of faster ablation time and higher temperatures, which could translate to successful treatment of larger tumours $[5,6]$. Emerging studies have demonstrated the effectiveness of MWA in the treatment of T1b RCCs, with high technical success $[7,8]$.

Non-target organ injury is a dreaded complication for any thermal ablation technique. This is especially relevant

Correspondence address:

Dr. Ronald Arellano, Department of Radiology, Division of Interventional Radiology, Massachusetts General Hospital, 55 Fruit St., 02114, Boston, United States, e-mail: rarellano@mgh.harvard.edu

Authors' contribution:

A Study design · B Data collection · C Statistical analysis · D Data interpretation · E Manuscript preparation · F Literature search · G Funds collection 
for thermal ablation adjacent to the ureter, where ureteral injury can result in devastating consequences of urinoma, ureteral stricture, and hydronephrosis. To mitigate this risk, several authors have described the technique of ureteral cooling with pyeloperfusion as an adjunctive technique during RFA for RCC $[9,10]$.

In contrast to RFA, which generates frictional heat via ionic agitation, MWA achieves tumouricidal temperatures through agitation of water molecules. As a result, MWA could theoretically increase the risk of thermal injury during MWA for RCC in proximity to the ureter. The purpose of this study was to present the preliminary outcomes of computed tomography (CT)-guided MWA with adjunctive pyeloperfusion in the treatment of centrally located RCCs.

\section{Material and methods}

\section{Patients}

The Institutional Review Board approved this Health Insurance Portability and Accountability Act-compliant retrospective analysis. An institutional database was reviewed to retrospectively identify patients with biopsyproven RCC treated with MWA between January 2016 and September 2017. The patients were then stratified into two cohorts: one cohort consisted of patients with RCC, who were treated with adjunctive pyeloperfusion; the

Table 1. Patient and tumour characteristics for pyeloperfusion and nonpyeloperfusion groups

\begin{tabular}{|c|c|c|c|}
\hline Characteristic & $\begin{array}{l}\text { Pyeloperfused } \\
\text { group }\end{array}$ & $\begin{array}{c}\text { Non- } \\
\text { pyeloperfused } \\
\text { group }\end{array}$ & $p$-value \\
\hline Number of patients & 9 & 9 & \\
\hline Age mean $( \pm S D)$ & $69.78( \pm 10.61)$ & $69.22( \pm 10.52)$ & 0.91 \\
\hline \multicolumn{4}{|l|}{ Gender (\%) } \\
\hline Male & 8/9 (89) & $7 / 9(77)$ & \multirow[t]{2}{*}{0.50} \\
\hline Female & $1 / 9(11)$ & $2 / 9(22)$ & \\
\hline Size of tumour (mm) & $32.78( \pm 12.63)$ & $29.22( \pm 8.05)$ & 0.41 \\
\hline \multicolumn{4}{|l|}{ Tumour polarity (\%) } \\
\hline Upper & $1 / 9(11)$ & $2 / 9(22)$ & \multirow[t]{3}{*}{0.33} \\
\hline Interpolar & $2 / 9(22)$ & $0 / 9(0)$ & \\
\hline Medial lower & $6 / 9(66)$ & $7 / 9(77)$ & \\
\hline \multicolumn{4}{|l|}{ Tumour location (\%) } \\
\hline Parenchymal & $4 / 9(44)$ & $4 / 9(44)$ & \multirow[t]{3}{*}{0.11} \\
\hline Exophytic & $2 / 9(22)$ & $5 / 9(55)$ & \\
\hline Mixed & $3 / 9(33)$ & $0 / 9(0)$ & \\
\hline $\begin{array}{l}\text { Distance to ureter } \\
( \pm S D)\end{array}$ & $18.44( \pm 12.63)$ & $23.22( \pm 12.71)$ & 0.43 \\
\hline
\end{tabular}

second cohort consisted of patients with RCC, who were matched based on tumour characteristics with the former group, and who were treated without pyeloperfusion. Selection of patients for either group was based on the interventional radiologist's clinical judgement, and the size and location of the tumour. Technical success was defined as completion of the planned procedures. Treatment outcomes were assessing by contrast material-enhanced CT or magnetic resonance imaging (MRI) at one month postMWA and then at six-month intervals thereafter. Collected data were evaluated for technical success, primary efficacy, secondary efficacy, renal function and duration, and number of procedures. The choice of pyeloperfusion was made on a case-by-case basis by an interventional radiologist considering centrally located tumours or tumours arising from the medial lower pole of the kidney and the risk of thermal injury to the ureter. The distance between the tumour and ureter and tumour size were measured on axial imaging. Locations of the tumours were categorised as exophytic, parenchymal, and mixed. Pre- and post-serum creatinine levels were measured. Patient and tumour characteristics are presented in Table 1.

\section{Procedure}

All ablations were performed with curative intent. The diagnosis of RCC was previously established by percutaneous biopsy. All ablations were performed with general anaesthesia or with intravenous procedural sedation. Computed tomography guidance (16-slice Lightspeed; GE Medical Systems, Madison, Wisconsin) was used for all procedures.

When critical non-target organs were adjacent to the targeted tumours, hydrodissection was performed by instillation of contrast-laced normal saline (10 cc Isovue mixed with $500 \mathrm{cc} 0.9 \%$ normal saline) to displace structures away from the kidney (Figure 1).

Pyeloperfusion was performed in nine patients whose tumours were within $2 \mathrm{~cm}$ of the proximal ureter. Immediately prior to the procedure, a urologist placed a $5 \mathrm{~F}$ open-ended ureteral stent (Pollack Open-End Flexi-Tip Ureteral Catheter; Cook Urological, Inc., Spencer, Indiana) into the ipsilateral ureter (Figure 2) with the distal end of the stent placed in the ipsilateral renal pelvis. The proximal end was externalised through the meatus and attached to a $1000 \mathrm{cc}$ bag of $0.9 \%$ normal saline and drip infused at approximately $1 \mathrm{drop} / \mathrm{sec}$.

At the end of the treatment a CT was performed to assess immediate complications. And immediately after the procedure the stent was removed. Surveillance imaging was done with CT and MRI with intervals of 1, 6, and 12 months and then annually to determine the presence of residual disease or collecting system injury. Complications were classified with the Clavien-Dindo system [11]. Repeat ablation was done if residual disease or new tumour was detected in surveillance imaging. 

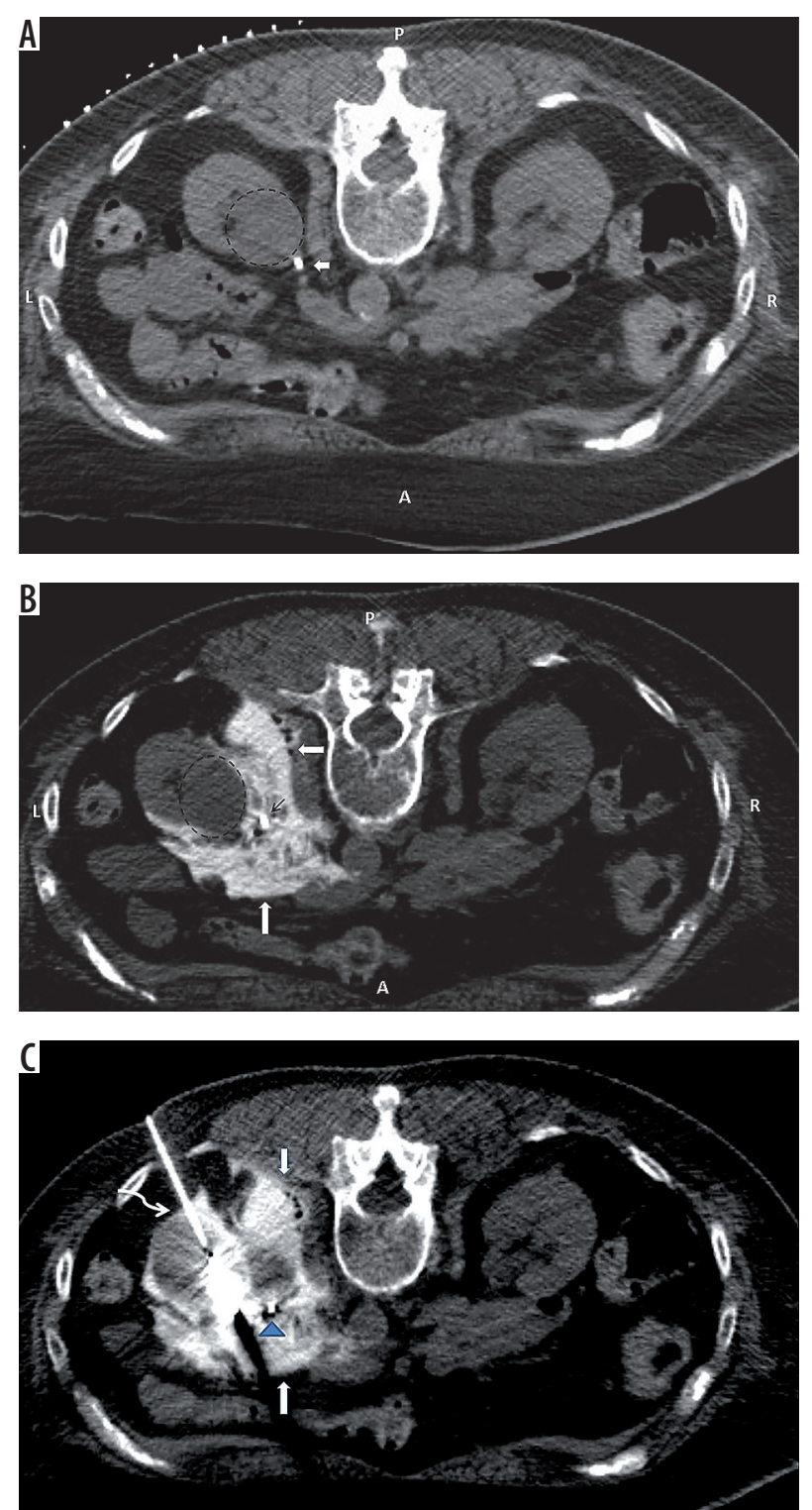

Figure 1. A) Pre-procedure computed tomography after placing a ureteral stent (arrow), demonstrating proximity of the ureter to the tumour (dashed circle). B) The ureter (narrow arrow) is displaced from the surface of the tumour (dashed circle) by instillation of diluted contrast (thick arrow). C) Microwave ablation of the tumour after pyeloperfusion (blue arrowhead) and hydrodissection (arrows) of the ureter; curved arrow shows ablation antenna

\section{Assessment of treatment response}

Assessment of the procedure was based on previously reported criteria [10]. Technical success was defined as successful completion of the planned ablation. Residual tumour was defined as persistent tumoural enhancement on CT or MRI on the first follow-up scan after the procedure. Primary efficacy and secondary efficacy were defined as percentage of tumours eradicated in first and second ablation sessions, respectively.

\section{Data collection and analysis}

Clinical and pathological features of the tumours were recorded and analysed retrospectively. Data for continu-
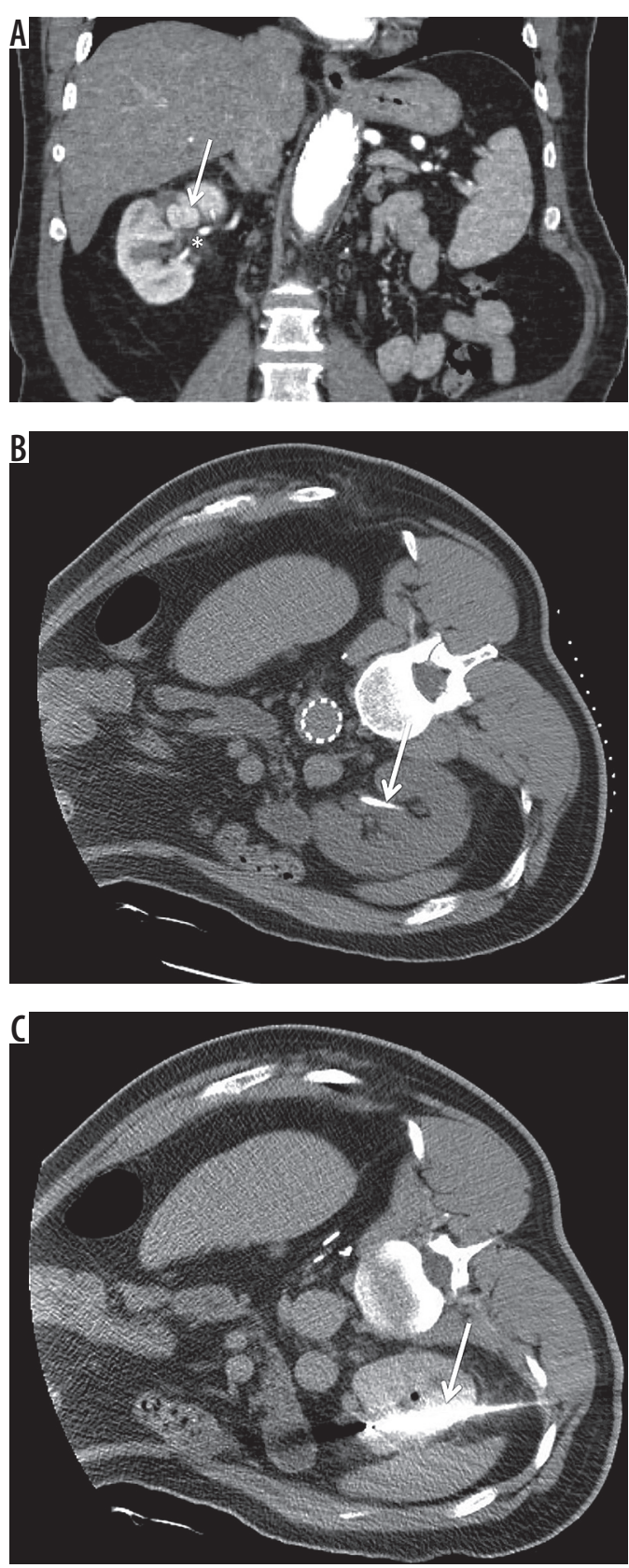

Figure 2. A) White arrow indicates central tumour; asterisk indicates renal pelvis. B) White arrow indicates stent for pyeloperfusion. C) White needle indicates microwave ablation antenna

ous variables were expressed as absolute numbers (mean $\pm \mathrm{SD}$ ). Paired $t$-test was used to compare means of continuous variables and Fisher exact test for comparison of percentage differences between different groups. A $p$-value $<0.05$ was considered statically significant. All statistical analyses were performed by using statistical software (SPSS 16.0; SPSS, Chicago, IL).

\section{Results}

This was an IRB approved study with waiver of patients consent (protocol number is 2018P001233). Eighteen biopsy-proven RCCs in 18 patients were treated with 
Table 2. Comparison of outcomes between pyeloperfusion and nonpyeloperfusion groups

\begin{tabular}{|c|c|c|c|}
\hline Efficacy measure & $\begin{array}{l}\text { Pyeloperfused } \\
\text { group }\end{array}$ & $\begin{array}{c}\text { Non- } \\
\text { pyeloperfused } \\
\text { group (\%) }\end{array}$ & $p$-value \\
\hline Residual disease (\%) & 1/9 (11\%) & $0 / 9(0)$ & 1.00 \\
\hline Primary efficacy (\%) & 8/9 (88) & $9 / 9(100)$ & 1.00 \\
\hline Secondary efficacy & $2 / 2(100)$ & 0 & \\
\hline \multicolumn{4}{|l|}{ Creatinine (\%)* } \\
\hline Before & $1.44( \pm 0.49)$ & $2.16( \pm 1.54)$ & \\
\hline After & $1.5( \pm 0.48)$ & $2.15( \pm 1.54)$ & \\
\hline $\begin{array}{l}\text { Duration of the } \\
\text { procedure }( \pm S D)^{* *}\end{array}$ & $144( \pm 35.34)$ & $95.5( \pm 34.04)$ & 0.004 \\
\hline $\begin{array}{l}\text { Number of ablative } \\
\text { sessions }\end{array}$ & 10 & 9 & \\
\hline $\begin{array}{l}\text { Overall } \\
\text { complication (\%) }\end{array}$ & $2 / 9(22)$ & $1 / 9(11)$ & 1.00 \\
\hline
\end{tabular}

*There was no significant difference in serum creatinine pre- and post-ablation for either group after and before the procedure in the pyeloperfused group or in the non-pyeloperfused group **Significant difference was because of the time required for stenting for pyeloperfusion

19 sessions of MWA. The mean age of the patients was $69 \pm 2.4$ years. The study patients were predominantly male (84\%). The tumour locations were classified as
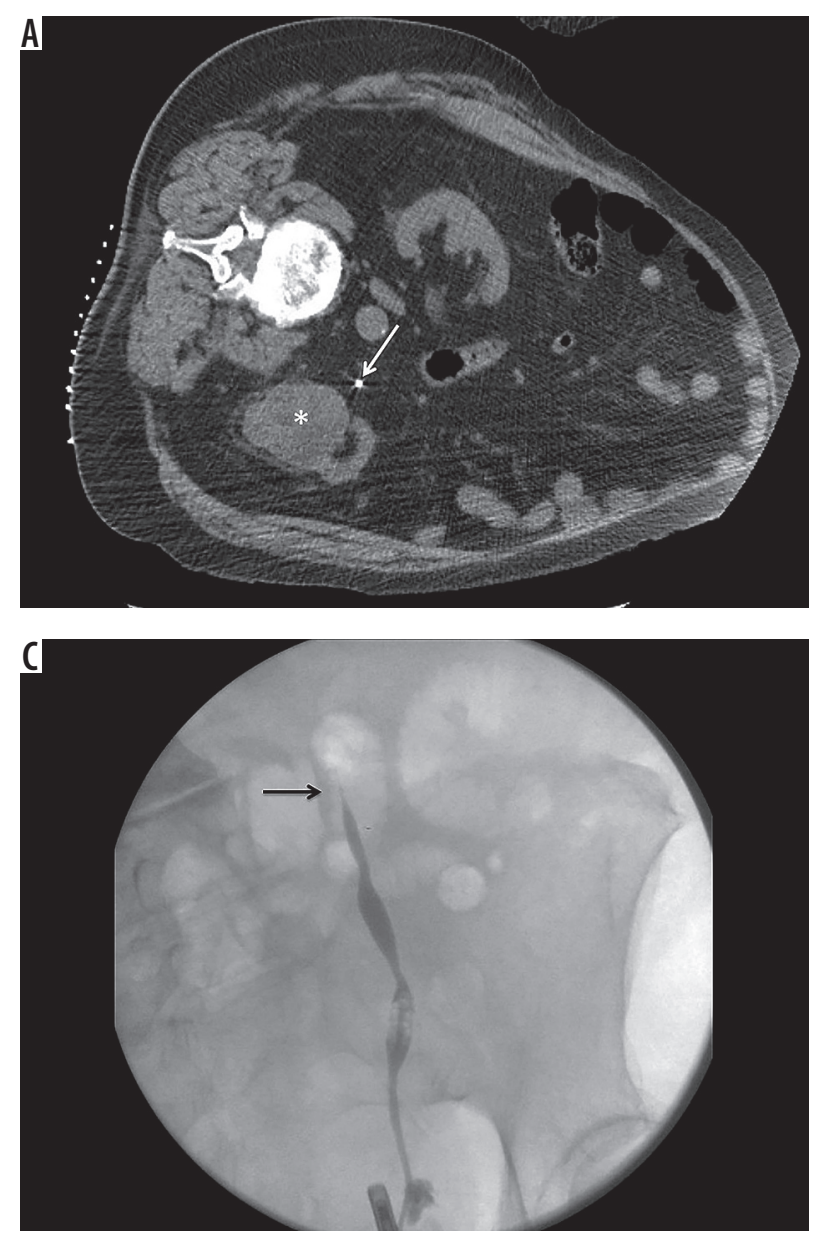

parenchymal $(n=8,44 \%)$, exophytic $(n=7,38 \%)$, and mixed $(n=3,16 \%)$. Mean tumour diameter was $31 \mathrm{~mm}$ ( $\pm 2.2 \mathrm{~mm}$ ). The average distance between ureter and the tumours in the pyeloperfused versus the non-pyeloperfused groups was 18.44 and $23.22(p=0.43)$, respectively, as summarised in Table 1. Hydrodissection was performed for two patients to displace the kidney from the psoas muscle and to displace the colon away from the kidney. The tumour polarity of this study is composed of $13(72 \%)$ medial lower tumours, three (16\%) upper, and two $(11 \%)$ interpolar tumours. The mean procedure time was 118 minutes. The average follow-up period was 180 days. Nine of 18 (50\%) patients had more than six months of follow-up. Mean pre- and post-creatinine values for the entire study were 1.80 and $1.99 \mathrm{mg} / \mathrm{dl}$, respectively $(p=0.4)$. The treatment outcomes for pyeloperfused and non-pyeloperfused groups are presented in Table 2.

Technical success was achieved for all patients. One pyeloperfused patient had residual disease on the onemonth follow-up, which was treated by repeat ablation. There were two grade 1 complications, including one small retroperitoneal haematoma and one small haematoma with concurrent pneumothorax. Both of these minor complications were treated conservatively.

There was one grade 2 complication, which consisted of ureteral stricture in a patient treated with pyeloperfu-

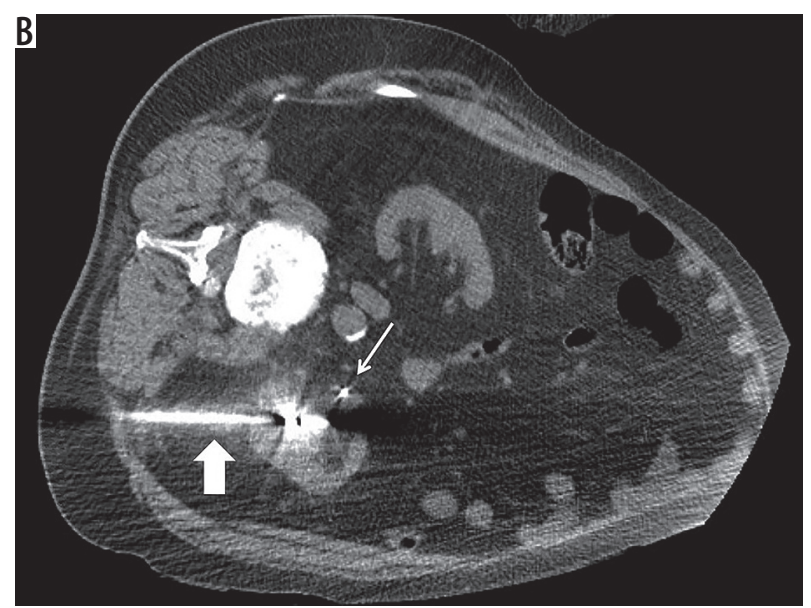

Figure 3. A) Axial unenhanced computed tomography (CT) scan of the abdomen at the time of ablation. Patient is in the left lateral decubitus position. White arrow indicates stent in the left ureter. White asterisk indicates renal cell carcinoma. B) Axial unenhanced (T scan of the abdomen at the time of ablation. Patient is in the left lateral decubitus position. White thin arrow indicates stent in the left ureter. White thick arrow indicates microwave ablation antenna. At the conclusion of the procedure, it was noted that the tubing connecting the bag of $0.9 \%$ normal saline to the ureteral stent became disconnected. As a result, it was likely that there was effective pyeloperfusion during treatment. C) Fluoroscopic images taken at the time of left retrograde pyelogram that demonstrate proximal ureteral stricture (black arrow) 
sion. The tumour was $55 \mathrm{~mm}$ in size and was located within $10 \mathrm{~mm}$ of the ureter (Figure 3). At the conclusion of the procedure, the ureteral stent was found to be disconnected from the $0.9 \%$ normal saline used for the drip infusion. Thus, we assume that, despite adequate positioning of the ureteral stent, pyeloperfusion was effectively not performed during the procedure. The patient ended up having a urological surgery to resolve the stricture.

Technical success was $100 \%$ for this study. Tumour location, polarity, and size were not identified as independently predictive of primary success in MWA (Table 3).

\section{Discussion}

Microwave ablation is a thermal ablative method that is used to treat liver and renal tumours. Its mechanism of action is based on electromagnetic waves that produce heat by agitation of water molecules, which leads to coagulation necrosis of the tissue and has a similar effectiveness profile compared to radiofrequency ablation $[6,12,13]$.

Because water is the main component of urine, there is a theoretically risk that MWA with pyeloperfusion may expose the ureter to increased risk of thermal injury. The results of this study show that with pyeloperfusion, MWA can be used to safely and effectively treat tumours near the renal pelvis and proximal ureter. The one case in our series of ureteral stricture that developed after MWA of a T1b tumour was probably the result of thermal injury to the ureter in the setting of pyeloperfusion failure due to a technical malfunction of the pyeloperfusion apparatus.

Location of the tumour is an important factor in the prediction of success and complication rate. Gervais et al. [14] showed that $25 \%$ of patients who underwent RF ablation for tumours within $1 \mathrm{~cm}$ of the ureter had ureteral stricture, whereas stricture resulted in $10 \%$ of the tumours within 1-2 cm of the ureter.

Pyeloperfusion is an adjunctive manoeuvre that has been shown to protect the ureter from thermal injury during radiofrequency ablation. Dai et al. [10] assessed the efficacy of RF ablation with pyeloperfusion in the management of central renal tumours and showed that compared to previous works $[14,15]$ the overall efficacy of the technique was improved after adding pyeloperfusion as an adjunct technique. There are two available studies demonstrating the clinical efficacy and safety of pyeloperfusion with RF ablation $[9,10]$. While pyeloperfusion with RFA has demonstrated safety, there are currently no reports of effectiveness of pyeloperfusion with MWA to treat RCC near the ureter or renal pelvis. Technical success was achieved for all patients in our study groups. Primary efficacy was achieved for $89 \%$ of pyeloperfused patients, which is higher compared to the results on RF and pyeloperfusion $[9,10]$, and the overall success rate of the study was $94 \%$, which is comparable with other initial report on MWA [8]. Schmit et al. [16] showed that
Table 3. Ablation outcomes based on tumour characteristics

\begin{tabular}{|c|c|c|c|}
\hline Characteristic & Number (\%) & Primary efficacy & $p$-value \\
\hline \multicolumn{4}{|l|}{ Size } \\
\hline$\geq 3 \mathrm{~mm}$ & $11 / 18(61)$ & $10 / 11(90)$ & \multirow[t]{2}{*}{1.00} \\
\hline$<3 \mathrm{~mm}$ & 7/18 (38) & $7 / 7(100)$ & \\
\hline \multicolumn{4}{|l|}{ Location } \\
\hline Exophytic & 8/18 (44) & $7 / 8(87)$ & \multirow[t]{3}{*}{0.44} \\
\hline Parenchymal & 7/18 (38) & $7 / 7(100)$ & \\
\hline Mixed & $3 / 18(16)$ & $3 / 3(100)$ & \\
\hline \multicolumn{4}{|l|}{ Polarity } \\
\hline Mid pole & 2/18 (11) & $2 / 2(100)$ & \multirow[t]{3}{*}{1.00} \\
\hline Upper & $3 / 18(16)$ & $3 / 3(100)$ & \\
\hline Lower & $13 / 18(72)$ & $12 / 13(92)$ & \\
\hline \multicolumn{4}{|c|}{ Distance to ureter } \\
\hline$\geq 20 \mathrm{~mm}$ & $10 / 18(55)$ & $10 / 10(100)$ & \multirow[t]{2}{*}{0.44} \\
\hline$<20 \mathrm{~mm}$ & $8 / 18(44)$ & $7 / 8(87)$ & \\
\hline \multicolumn{4}{|l|}{ Tumoural side } \\
\hline Left & $13 / 18(72)$ & $12 / 13(92)$ & \multirow[t]{2}{*}{1.00} \\
\hline Right & $5 / 18(27)$ & $5 / 5(100)$ & \\
\hline
\end{tabular}

the primary effectiveness of radiofrequency ablation is limited to exophytic and small $(<3)$ tumours; however, we found no relation between ablation outcome and tumour complexity factors, including size, location, and polarity independently.

There were only two grade 1 (Clavien-Dindo 1) complications and one grade 2 complication, which was most likely due to pyeloperfusion malfunction. The complication rate in our study was similar to previous reports that assessed the efficacy of MWA in the treatment of renal tumours with larger groups of patients $[7,8]$. Cantwell et al. [9] reported $19 \mathrm{RF}$ ablations with adjunctive pyeloperfusion without any ureteral stricture. In our study the only case of ureteral stricture was a patient with malfunctioned pyeloperfusion. Dong et al. [17] demonstrated in their study that haematoma is more prevalent in exophytic tumours, while urinary tract complications occur mainly in endophytic tumours. One study by Castle et al. [18] reported a $20 \%$ intraoperative and $40 \%$ postoperative complication rate in MWA of RCCs, which is probably due to the involvement of the urinary tract in half of the patients, However, the efficacy of pyeloperfusion in protecting the urinary tract was not evaluated in this study.

Similarly to the earlier study by Dong et al. [17], we found no significant different between renal function after and before ablation. In another study Wah et al. [19] showed that there was $3.1 \%$ worsening of renal function after performing RF for lower lobe renal tumours. 


\section{Limitations}

This study has several limitations. It is a non-randomised retrospective analysis of a small cohort of patients. While the results are encouraging, the small patient population limits the generalisability of the results. A larger cohort would be helpful to evaluate the validity of our results. The study is also limited by a short overall follow-up period. This is due, in part, to the relatively short time that MWA has been used at our institution. We hope to report long-term results as they become available. The results of this study suggest that microwave ablation of centrally located tumours with pyeloperfusion is safe and effective and should be considered as an alternative thermal ablation modality to RFA and cryoablation for the treatment of RCC.

\section{Conclusions}

Hydrodissection is a useful adjunctive technique for MWA of RCCs to keep adjacent structures from untargeted thermal injury. Pyeloperfusion is a technique to protect the ureter from thermal injury during MWA of lower renal pole tumours. MWA is a safe and effective treatment for RCC.

\section{Conflict of interest}

The authors report no conflict of interest.

\section{References}

1. Gervais DA, McGovern FJ, Arellano RS, et al. Radiofrequency ablation of renal cell carcinoma: Part 1. Indications, results, and role in patient management over a 6-year period and ablation of 100 tumors. Am J Roentgenol 2005; 185: 64-71.

2. Campbell SC, Novick AC, Belldegrun A, et al. Guideline for management of the clinical T1 renal mass. J Urol 2009; 182: 1271-1279.

3. El Dib R, Touma NJ, Kapoor A. Cryoablation vs radiofrequency ablation for the treatment of renal cell carcinoma: a meta-analysis of case series studies. BJU Int 2012; 110: 510-516.

4. Thompson RH, Atwell T, Schmit G, et al. Comparison of partial nephrectomy and percutaneous ablation for cT1 renal masses. Eur Urol 2015; 67: 252-259.

5. Yu J, Liang P, Yu X, et al. A comparison of microwave ablation and bipolar radiofrequency ablation both with an internally cooled probe: results in ex vivo and in vivo porcine livers. Eur J Radiol 2011; 79: 124-130.

6. Carrafiello G, Lagana D, Mangini M, et al. Microwave tumors ablation: principles, clinical applications and review of preliminary experiences. Int J Surg 2008; 1: 65-69.

7. Liang P, Wang Y, Zhang D, et al. Ultrasound guided percutaneous microwave ablation for small renal cancer: initial experience. J Urol 2008; 180: 844-848

8. Yu J, Liang P, Yu XL, et al. US-guided percutaneous microwave ablation of renal cell carcinoma: intermediate-term results. Radiology 2012; 263: 900-908.

9. Cantwell CP, Wah TM, Gervais DA, et al. Protecting the ureter during radiofrequency ablation of renal cell cancer: a pilot study of retrograde pyeloperfusion wit cooled dextrose 5\% in water. Vasc Interv Radiol 2008; 19: 1034-1040.

10. Dai $Y$, Covarrubias D, Uppot R, et al. Image-guided percutaneous radiofrequency ablation of central renal cell carcinoma: assessment of clinical efficacy and safety in 31 tumors. J Vasc Interv Radiol 2017; 28: 1643-1650.

11. Dindo D, Demartines N, Clavien PA. Classification of surgical complications: a new proposal with evaluation in a cohort of $6336 \mathrm{pa}-$ tients and results of a survey. Ann Surg 2004; 240: 205-213.

12. Wingo MS, Leveillee RJ. Central and deep renal tumors can be effectively ablated: radiofrequency ablation outcomes with fiberoptic peripheral temperature monitoring. J Endourol 2008; 22: 1261-1267.

13. Wright AS, Sampson LA, Warner TF, et al. Radiofrequency versus microwave ablation in a hepatic porcine model. Radiology 2005; 236: 132-139.

14. Gervais DA, McGovern FJ, Arellano RS, et al. Radiofrequency ablation of renal cell carcinoma: part 1, Indications, results, and role in patient management over a 6-year period and ablation of 100 tumors. AJR Am J Roentgenol 2005; 185: 64-71.

15. Takaki H, Yamakado K, Soga N, et al. Midterm results of radiofrequency ablation versus nephrectomy for T1a renal cell carcinoma. Jpn J Radiol 2010; 28: 460-468.

16. Schmit GD, Thompson RH, Kurup AN, et al. Usefulness of R.E.N.A.L. nephrometry scoring system for predicting outcomes and complications of percutaneous ablation of 751 renal tumors. J Urol 2013; 189: 30-35.

17. Dong X, Yu X, Yu M, et al. Complications of ultrasound-guided percutaneous microwave ablation of renal cell carcinoma. Onco Targets Ther 2016; 9: 5903-5909.

18. Castle SM, Salas N, Leveillee RJ. Initial experience using microwave ablation therapy for renal tumor treatment: 18-month follow-up. Urology 2011; 77: 792-797.

19. Wah T, Irving H, Gregory W, et al. Radiofrequency ablation (RFA) of renal cell carcinoma (RCC): experience in 200 tumours. BJU Int 2014; 113: 416-428. 